\title{
Simulation of a Ridge-Type Semiconductor Laser with Horizontal Coupling of Lateral Modes
}

\author{
Guowei Chai and Takahiro Numai \\ Graduate School of Science and Engineering, Ritsumeikan University \\ 1-1-1 Noji-Higashi, Kusatsu, Shiga 525-8577, Japan \\ Phone: +81-77-561-5161 E-mail: numai@se.ritsumei.ac.jp
}

\section{Introduction}

High power 980-nm semiconductor lasers are indispensable to pump erbium doped optical fiber amplifiers [1]. Generally, 980-nm semiconductor lasers have ridge structures to confine lateral modes. This reason is as follows: the cladding layers of 980-nm semiconductor lasers are AlGaAs layers. When buried structures are fabricated, AlGaAs layers are etched selectively before epitaxial regrowth to bury a mesa. During etching process of AlGaAs layers, AlGaAs layers are oxidized easily, and electrical and optical characteristics are highly degraded. To avoid these degradations, ridge structures are adopted. In the ridge structures, higherorder lateral modes as well as the fundamental lateral mode are confined. As a result, with an increase in injected current, higher-order lateral modes lase; kinks appear in their current versus lightoutput $(I-L)$ curves [2]. The light output where a kink appears is called kink level. Below the kink level, the fundamental lateral mode is obtained; above the kink level, higher order lateral modes are oscillated. Light coupling efficiency to the single mode fiber of the higher-order lateral modes is much lower than that of the fundamental lateral mode. As a result, to obtain high fiber-coupled optical power, high kink levels or no kinks are required. To date, to increase the kink levels, coupling of the optical field to the lossy metal layers outside the ridge [3], highly resistive regions in both sides of ridge stripe [4], and incorporation of a graded Vshape layer [5] were investigated. To increase the kink level further, a ridge structure with optical antiguiding layers [6], [7] and a ridge structure with separate confinement of carriers and horizontal transverse mode [8] have been proposed. However, kinks have always appeared.

In this paper, a ridge-type semiconductor laser with horizontal coupling of lateral modes is proposed to make the fabrication process simple, and increase the kink level. In this semiconductor laser, a groove is formed in the center of the mesa, and the lateral modes of the laser beams are determined by the coupling of the lateral modes confined by the two ridges, which are formed in the mesa.

From simulations, it is found that kinks do not appear by optimizing the width and depth of the groove.

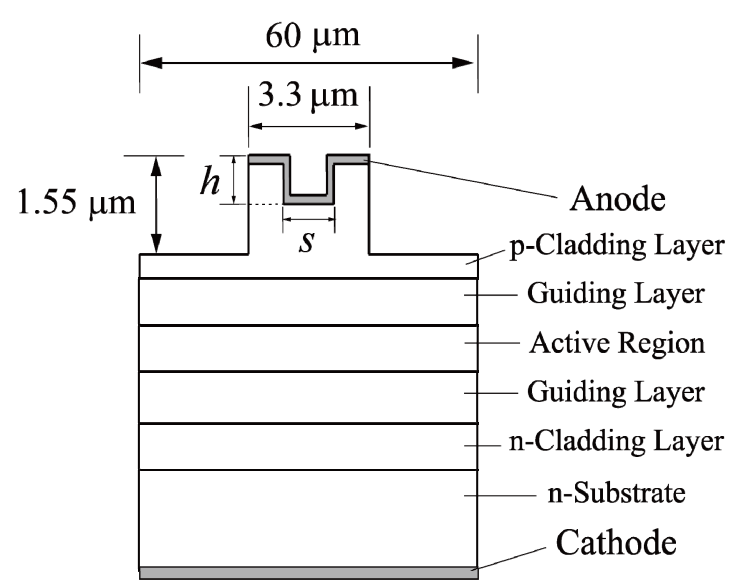

Fig. 1 Schematic cross-sectional view of a proposed ridge structure with horizontal coupling of lateral modes. A groove is formed in the center of the mesa and two ridges are formed. Here, $S$ is the width of the groove in the mesa and the space between the two ridges; $h$ is the depth of the groove in the mesa and the height of the two ridges measured from the bottom of the groove.

\section{Laser Structures and Simulations}

Figure 1 shows a schematic cross-sectional view of a proposed ridge structure with horizontal coupling of lateral modes. A grove is formed in the center of the mesa and two ridges are formed. These two ridges confine lateral modes, and the lateral modes are coupled through the groove. It is expected that the coupling of the fundamental lateral modes for each ridge becomes higher than the coupling of the higher-order lateral modes for each ridge. In Fig. 1, $S$ is the width of the groove and the space between the two ridges; $h$ is the depth of the groove and the height of the two ridges measured from the bottom of the groove. The mesa is 1.55 $\mu \mathrm{m}$ high and $3.3 \mu \mathrm{m}$ wide. The base is $60 \mu \mathrm{m}$ wide, and the cavity is $1200 \mu \mathrm{m}$ long. Reflectivities of the front and rear facets are 2 and $90 \%$, respectively.

Layer parameters such as band gap energy, refractive index, thickness, electron effective mass, hole effective mass, and doping concentration are the same as those described in Refs. 6-8. Lasing characteristics are simulated by using a device simulation software ATLAS (Silvaco) in which Poisson's equations and Helmholtz equation are solved with a finite element method. 


\section{NUSOD 2013}

\section{Simulation Results and Discussions}

Figure 2 shows injected current versus light output characteristics. The parameter is the width $S$ of the groove in the mesa. The depth $h$ of the groove in the mesa is $1000 \mu \mathrm{m}$. The kink level depends on the width $S$ of the groove, and the highest kink level is obtained at $S=1.32 \mu \mathrm{m}$.

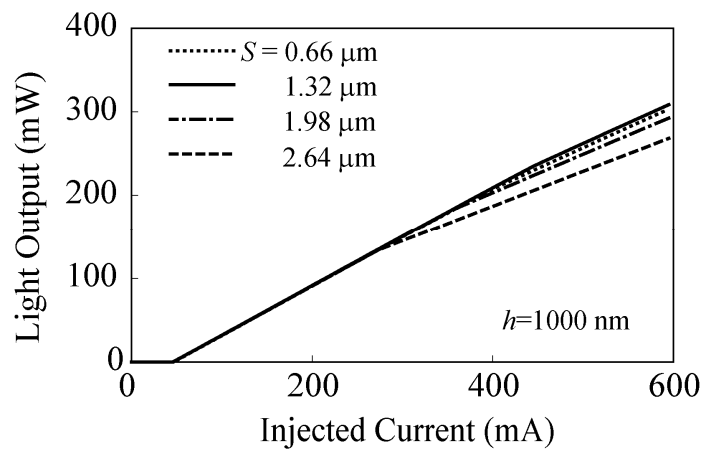

Fig.2 Injected current versus light output curves. The highest kink level is obtained at $S=1.32 \mu \mathrm{m}$.

Figure 3 shows kink levels as a function of the width $S$ of the groove in the mesa. The parameter is the depth $h$ of the groove in the mesa. With an increase in the depth $h$ of the groove, the kink levels increase. For $h \leq 1000 \mathrm{~nm}$, the kink levels are the highest at $S=1.32 \mu \mathrm{m}$. For $h=1200 \mathrm{~nm}$, kinks do not appear in $S \leq 1.98 \mu \mathrm{m}$ up to the injected current of $2 \mathrm{~A}$.

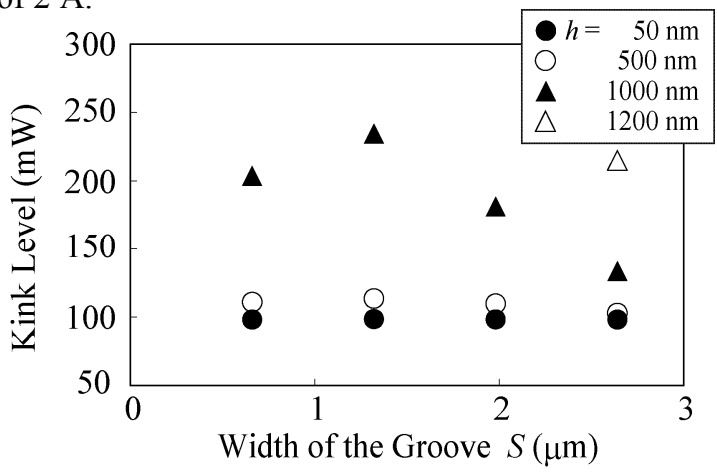

Fig.3 Kink levels as a function of the width $S$ of the groove in the mesa. With an increase in the depth $h$ of the groove, the kink levels increase. For $h=1200 \mathrm{~nm}$, kinks do not appear in $S \leq 1.98 \mu \mathrm{m}$.

Figure 4 shows the threshold current $I_{\text {th }}$ for the fundamental lateral mode as a function of the width $S$ of the groove in the mesa. The parameter is the depth $h$ of the groove in the mesa. The threshold current decreases with an increase in the depth $h$ of the groove in the mesa. For $h \geq 500 \mathrm{~nm}$, the threshold current decreases with an increase in the width $S$ of the groove in the mesa. The lowest threshold current for kink-free operation is obtained at $S$ $=1.98 \mu \mathrm{m}$ and $h=1200 \mathrm{~nm}$, and its value is $50.2 \mathrm{~mA}$, which is slightly larger than the simulated lowest value of $46.3 \mathrm{~mA}$ in Ref.8. It should be noted that kinks do not appear in the present paper while kinks appeared in Ref. 8.

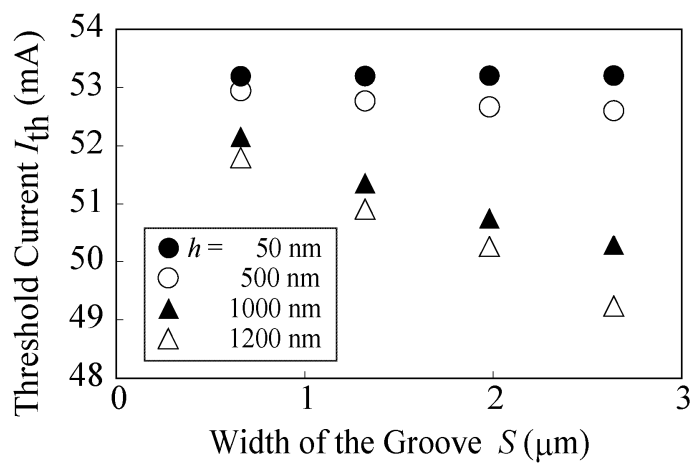

Fig.4 Threshold current $I_{\text {th }}$ for the fundamental lateral mode as a function of the width $S$ of the groove in the mesa. The threshold current decreases with an increase in the depth $h$ of the groove in the mesa. For $h \geq 500 \mathrm{~nm}$, the threshold current decreases with an increase in the width $S$ of the groove in the mesa.

\section{Conclusions}

To improve kink levels in ridge-type semiconductor lasers, a novel ridge structure with horizontal coupling of lateral modes by a groove in the mesa was proposed and simulated. It is found that kinks do not appear by optimizing the width and the depth of the groove in the center of the mesa.

\section{Acknowledgement}

This research was partially supported by the Japan Society for Science, Grant-in-Aid for Scientific Research (C) 24560429, 2012.

\section{References}

[1] C. S. Harder, L. Brovelli, H. P. Meier, and A. Oosenbrug, Proc. Optical Fiber Communication Conf. '97, February, FC1, 1997, p.350.

[2] M. F. C. Schemmann, C. J. van der Poel, B. A. H. van Bakel, H. P. M. M. Ambrosius, A. Valster, J. A. M. van den Heijkant, and G. A. Acket, Appl. Phys. Lett., vol.66, pp.920-922 (1995).

[3] M. Buda, H. H. Tan, L. Fu, L. Josyula, and C. Jagadish, IEEE Photonics Technol. Lett., vol.15, pp.16861688 ( 2003).

[4] M. Yuda, T. Hirono, A. Kozen, and C. Amano, IEEE J. Quantum Electron., vol.40, pp.1203-1207 (2004).

[5] B. Qiu, S. D. McDougall, X. Liu, G. Bacchin, and J. H. Marsh, IEEE J. Quantum Electron., vol.41, pp.1124-1130 (2005).

[6] N. Shomura, M. Fujimoto, and T. Numai, IEEE J. Quantum Electron., vol.44, pp.819-825 (2008).

[7] H. Yoshida and T. Numai, Jpn. J. Appl. Phys., vol.48, 082105, 2009.

[8] H. Kato, H. Yoshida, and T. Numai, Opt. and Quantum Electron, 2012. DOI 10.1007/s11082-012-9620-x 